\title{
A Proposed Typology of Sampled Material Within Electronic Dance MUSic ${ }^{1}$
}

\author{
$\bullet$ Feature Article $\longrightarrow$ \\ ROBERT RATCLIFFE \\ Manchester Metropolitan University (UK)
}

\begin{abstract}
The following article contains a proposed typology of sampled material within electronic dance music (EDM). The typology offers a system of classification that takes into account the sonic, musical and referential properties of sampled elements, while also considering the technical realisation of the material and the compositional intentions of the artist, producer or DJ. Illustrated with supporting examples drawn from a wide variety of artists and sub-genres, the article seeks to address the current lack of research on the subject of sample-based composition and production, and provides a framework for further discussion of EDM sampling practices. In addition, it demonstrates how concepts and terminology derived from the field of electroacoustic music can be successfully applied to the study and analysis of EDM, resulting in an expanded analytical and theoretical vocabulary.
\end{abstract}

KEYWORDS: EDM, sample, sampling, musical borrowing, production, composition, electroacoustic, mimesis, transcontextuality, spectromorphology, source bonding

ROBERT RATCLIFFE is an internationally recognised composer, sonic artist, EDM musicologist and performer. He completed a $\mathrm{PhD}$ in composition and musicology funded by the Arts and Humanities Research Council at Keele University, UK. He has developed a hybrid musicallanguage and compositional technique through the cross-fertilisation of art music and electronic dance music (EDM). His hybrid compositions have been performed and broadcast in over twenty-five countries worldwide, including presentations at international events such as ACMC, ICMC, L'espace du Son, NIME and Sonorities.

Dancecult: Journal of Electronic Dance Music Culture 6(1): 97-122 ISSN 1947-5403 @2014 Dancecult http://dj.dancecult.net DOI 10.12801/1947-5403.2014.06.01.06 


\section{INTRODUCTION}

There have been a number of attempts to categorise the sampling ${ }^{2}$ practices of art and popular electronic musics, examining both the materials and compositional techniques involved. ${ }^{3}$ Goodwin's taxonomy of sampling within popular production (1990) proposes three separate categories, based on the intent and motivations of the borrower, and the referential qualities of the material. Porcello also makes a distinction between forms of commercial music sampling, recognising three strands that differ in terms of technical implementation and intended mimetic function: mimetic/reproductive, manipulative and extractive (1991). In contrast, Cutler's review of "plunderphonic" applications in electronic art music (2000) identifies five categories, which take into account the compositional procedures involved and the properties of the borrowed elements, including size and recognisability. Although Cutler touches on popular music, a closer examination of sampling within vernacular forms is provided by Sanjek (1994) who differs from Goodwin and Porcello in his assertion of four strands, including a separate category for remixing. ${ }^{4}$ In addition, there are several introductory overviews of sampling practice (for instance Butler 2006; Cox 2007; Metzer 2003). Such descriptions tend to categorise according to sound type, making a distinction between the use of smaller fragments and extended samples without going into considerable detail or providing specific musical examples.

The musicological study of electronic dance music $(\mathrm{EDM})^{5}$ is a newly emerging area of research, and, to my knowledge, this article is the first detailed exposition of sample-based EDM. The proposed system of classification takes into consideration both the properties and the functionality of borrowed material, while providing additional information concerning the technical and compositional aspects of sampling. It is intended for the proposed typology to facilitate a better understanding of the materials and processes of EDM composition and lead to a more informed listening experience. The typology is illuminated by supporting examples sourced from interviews with EDM artists, producers and DJs operating in a variety of sub-genres. It also explores the use of concepts and terminology derived from electroacoustic music (in particular, the ideas of Simon Emmerson and Denis Smalley) to provide a descriptive vocabulary for EDM analysis. The main argument here is that there are many connections and commonalities that exist between EDM and electroacoustic music, as seen in the similarity of certain sound materials, and to some extent the compositional procedures and means of production, as directed by available technology. In the following text, specific links are identified in order to demonstrate the effectiveness and potential for a wider application of the electroacoustic device within EDM analysis, resulting in an expanded analytical and theoretical vocabulary.

The sampled materials of EDM can be grouped into four main categories: (A) short, isolated fragments; ${ }^{6}$ (B) loops and phrases; (C) larger elements; (D) transformed material. These categories can then be divided into further sub-categories depending on the musical function ${ }^{7}$ and referential ${ }^{8}$ qualities of the material. A visual representation of the typology is provided below (fig. 1). 


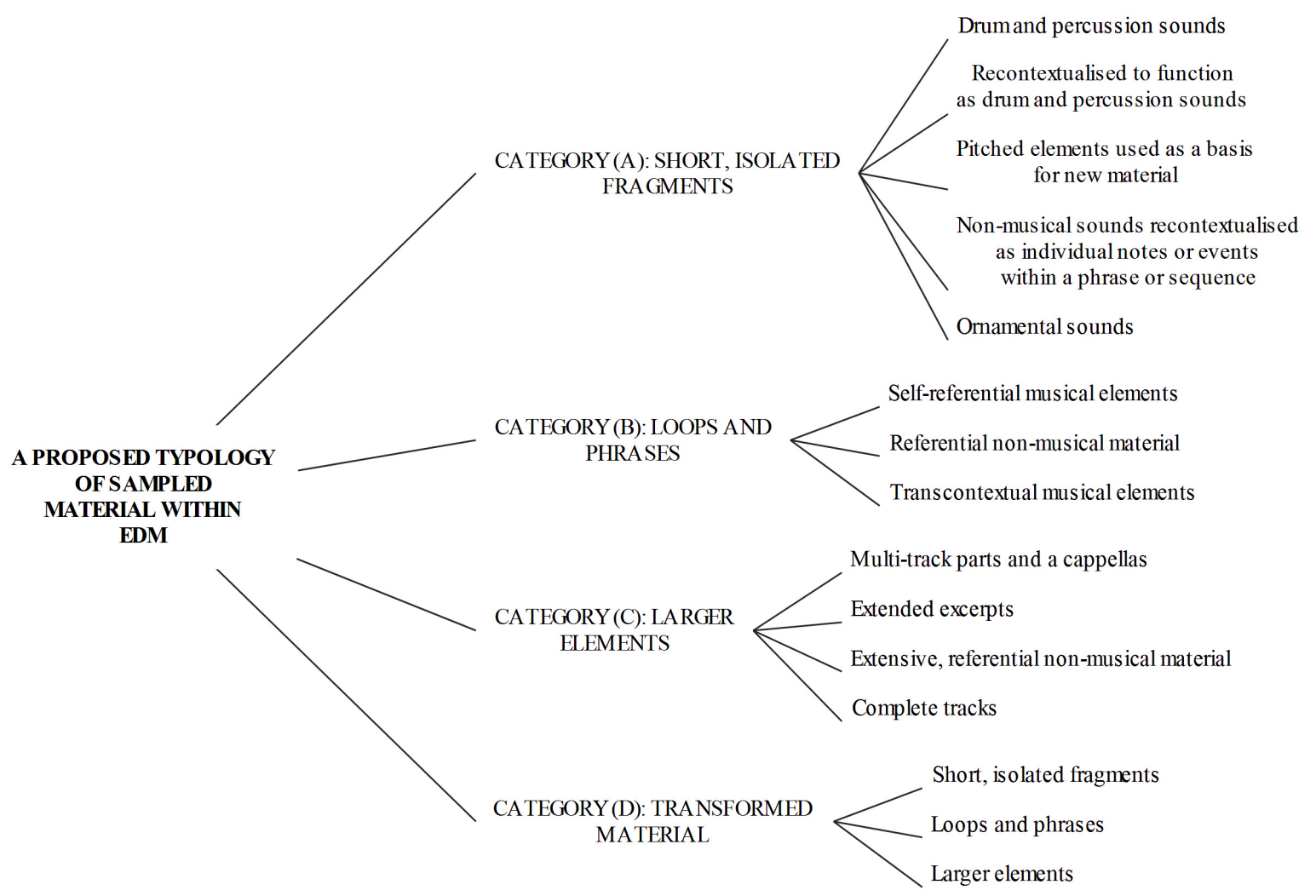

FigURE 1: A PROPOSED TYPOLOGY OF SAMPLED MATERIAL WITHIN EDM.

The first three categories of the typology are based on structural properties (or sample length), with the structural possibilities afforded by sound transformation also accounted for through the inclusion of a separate category for transformed material. The methodology developed provides an effective approach to sample classification, due to the way in which samples are incorporated within EDM-this often involves the selection and editing of material according to a specific duration or periodicity, whether it is a single drum hit, a phrase or pattern length, or an entire section of music. Sample length may also be predetermined by the specifications of the technology employed; for example, a sampling drum machine may offer a very short recording time or maximum sample length, as it is designed to work with percussion sounds. In contrast, performance samplers are optimised for working with loops and phrases, while software permits the use of larger elements. Older technology will also impose its own restrictions due to limited onboard storage.

\section{CATEgory (A): Short, Isolated Fragments}

\section{SHORT, ISOLATED FRAGMENTS: DRUM AND PERCUSSION SOUNDS}

The first sub-category consists of individual percussion sounds that are sampled for use within a rhythmic pattern or stream. Typically, this will involve samples collected from a multitude of sources, which are used as elements in a breakbeat or synthetic drum pattern. 
Individual samples may be layered ${ }^{9}$ against existing patterns and loops, or used to form a percussion "sample-bank" that can be controlled from a sequencer, as DJ Shadow elaborates:

I sample a kick, a snare and some hats, maybe from different places. Then I build up the loop on the sequencer. Sometimes it doesn't have the right vibe, so I might sample some atmosphere ${ }^{10}$ and drop that in between the kicks and snares just to make it feel live (cited in Scott 1998: 99).

A crucial, yet time-consuming part of this process involves the location and extraction of suitable source material, "much like (and often one and the same as) a DJ's practice of 'digging' for vinyl records” (Rodgers 2003: 318). The importance of collecting, critiquing and cataloguing source material was highlighted by Fatboy Slim (Norman Cook) in a description of the pre-compositional process behind his third album, Halfway Between the Gutter and the Stars (2000). Interestingly, Cook documents a process similar to that employed by electroacoustic composers who often collect and catalogue different sound sources along with extensive variations, according to different processes applied to the original (for example, reverb and delay):

I realised the starting point for me has always been source material. Y'know... samples. So I spent the next few weeks trawling round record fairs and second-hand shops, buying up crateloads of crappy albums for $25 \mathrm{p}$. Then it was just a question of sitting down and sifting through everything, finding the bits I liked, sticking them on to disk and cataloguing it all. I have all my bass riffs all set out in different bpms... my loops all chopped into kick, snare and hat... that kind of thing (cited in Scott 2001: 96).

\section{SHORT, ISOLATED FRAGMENTS: RECONTEXTUALISED TO FUNCTION AS DRUM AND}

\section{PERCUSSION SOUNDS}

The second sub-category contains sound fragments that are non-percussion-based (although they may contain gestural information ${ }^{11}$ associated with percussive sounding materials), ${ }^{12}$ which are recontextualised to function as drum and percussion elements. A common strategy involves the creation of a rhythmic (or "groove") template that is extracted from an existing breakbeat using specialised software, such as Propellerhead's ReCycle. ${ }^{13}$ This is achieved by analysing the performance characteristics of the sampled breakbeat (for example, note onset, duration and velocity information), and allows for the inherent performance properties of the sample to be maintained while replacing the original sounds with recontextualised elements. In an interview with Sound On Sound magazine, the breakcore ${ }^{14}$ producer Venetian Snares describes a similar process, whereby the performance characteristics of a breakbeat are used in conjunction with unconventional percussion sounds:

Well, yes, in a way they are breakbeats. I'm just not using traditional drum sounds a lot of the time. I prefer to create my own sounds from scratch. A lot of the time I'll take a breakbeat, originally played by a drummer on a traditional kit, and replace all the drum hits with my own sounds, then chop that up and construct the beats from 
that. One method is to open the breakbeat in Sound Forge ${ }^{15}$ and regionalise all of the individual hits, by hand - the auto-region function has never seemed accurate enough to me. Then I'll mute all the hits, but leave the regions still marked. Then I'll put my own drum sounds into the regions using the Mix function. It's great because you can layer a lot of sounds in the regions, just as a drum kit would have hats and snares and so on overlapping and interacting with each other. I can have the flow and nuances of real drums, but with whatever sounds I want. The possibilities are endless! (cited in Sellars 2002: 178)

In the track "Timber" (1997) (a politically-charged collaboration between Coldcut and Hexstatic) recontextualised sounds (associated with industrial $\operatorname{logging}^{16}$ ) are used mimetically ${ }^{17}$ to evoke images of deforestation. The design of the rhythmic material is particularly interesting, as the samples are sequenced and manipulated in order to simulate actions of cutting, chopping and sawing (an example of "syntactic mimesis"18 (Emmerson 1986)).

As suggested above, the ideas of electroacoustic composer and theorist Simon Emmerson may be helpful in discussing the mimetic aspects of sampled sound in EDM. His influential chapter "The Relation of Language to Materials" (1986) explores mimesis in electroacoustic music as part of a proposed continuum of musical discourse (aural and mimetic), which he relates to musical syntax (abstract and abstracted) in a survey of "approaches to language" (1986: 24).

\section{SHORT, ISOLATED FRAGMENTS: PITCHED ELEMENTS USED AS A BASIS FOR NEW} MUSICAL MATERIAL

The third sub-category contains pitched elements (for example, individual notes or chords) that are sampled and reconfigured to form new material. The motivations for working in this way may vary from the aesthetic, to the ethical or economic. For instance, if a musical phrase has been sampled for its spectral qualities, the application of sound transformation to disguise the origin of the source would remove or distort these properties and render the sample obsolete. As an alternative, EDM musicians have developed a variety of methods for generating similar material to help prevent source identification. One way to obscure the identity of the source, while retaining its spectral properties, is to extract individual notes or elements from the sample and to use these in the construction of a new musical phrase. ${ }^{19}$ This strategy is frequently employed by artists such as the Avalanches who altered their approach to working with sampled material:

The most basic change has been in what we see as sample-worthy in the first place. Instead of loops or grooves we've both amassed large collections of chords and notes which in the past we would have ignored. These are pieced together to slowly create small sections - our new loops and grooves - with which we can construct songs (cited in Pytlik 2002: 76). 
Another reason why EDM musicians dissect and recombine samples is because the use of larger, unmodified extracts is often viewed as uncreative or unoriginal. For DJ Shadow, constructing music from substantial, unaltered material is "an injustice to the art of sampling", and he is "strongly opposed to that way of working" (cited in Scott 1998: 99). It is ironic to find the notion of "authorship" nested within sampling practices, yet musicians such as DJ Shadow ${ }^{20}$ only gain a sense of ownership once the source material has been deconstructed and reconfigured to form something new. In the following example, he describes an approach similar to the Avalanches:

What I do is find a bassline with the right feel then I sample individual notes of that bassline, assign them to different pads on the $\mathrm{MPC}^{21}$ and restructure them to play a different tune (cited in Scott 1998: 99).

This approach features a gestural use of the sampler that is similar to playing a synthesizer or keyboard containing sample-based sounds (for example, Yamaha's AWM (Advanced Wave Memory) system). In this way, the sampler is used to facilitate a form of stored waveform synthesis. ${ }^{22}$ Typically, this will involve editing and looping a short sample, which is mapped across a keyboard (or other interface) and played/programmed to create new material. A notable example is the "reese" bass sample, which is a synthetic bass sound sampled" ${ }^{23}$ and replayed on innumerable jungle and drum 'n' bass records. The original sound has been traced to the Kevin Saunderson track "Just Want Another Chance" (1988) (released under the pseudonym Reese), and sampled on tracks such as "The Mutant Remix - Rollers Instinct" [DJ Trace remix] (1995) by T. Power (Reynolds 1999: 22 and 352).

\section{SHORT, ISOLATED FRAGMENTS: NON-MUSICAL SOUNDS RECONTEXTUALISED AS \\ INDIVIDUAL NOTES OR EVENTS WITHIN A PHRASE OR SEQUENCE}

The fourth sub-category contains musical material formed through the organisation of non-musical sound. More specifically, short samples derived from a non-musical object are recontextualised as individual components (notes or events) within a musical phrase or sequence. The material is produced through detailed sample editing (individual samples are often extracted from field recordings) and the use of a controlling sequencer to organise the various fragments into a phrase-level structure. Specific examples include the "chainsaw solo" ${ }^{4}$ of "Timber" (1997), and the more recent "Printer Jam"25 (2009) by Mistabishi, where samples of a computer printer are recontextualised to function as the synthetic sounds of drum ' $n$ ' bass. Through such examples we can see how EDM musicians explore the musical potential of non-musical materials in a similar way to composers of musique concrète, although recognition of the sound source is intended and often necessary in order to understand the meaning of the samples used. The connection between the use of these materials in EDM and musique concrète has been noted by others, and acknowledged by artists such as Matmos: 
... we started to make dance music patterns but have them play only these noisy, difficult, strange sounds. It was almost like the vocabulary was from industrial music and musique concrète, but the grammar was the cut-up of breakbeat techno (Daniel cited in Doyle 2004a: 78).

\section{SHORT, ISOLATED FRAGMENTS: ORNAMENTAL SOUNDS}

The fifth sub-category includes sound fragments that have an ornamental function, such as vocal hits, orchestral stabs, and a whole range of sonic embellishments. ${ }^{26}$ These can still have a mimetic ${ }^{27}$ quality in that they evoke a certain genre or historical production style: ${ }^{28}$

I like the idea of a drum coming from a different age and a different place with a different spirit. When you bring these things together, you get this weird chemistry. Like the trail of sound after a snare drum - that is the ambience of that room and what was going on in that room - all these weird, different feelings from different places (Tom Rowlands of the Chemical Brothers, cited in Micallef 2002).

Similarly, for the producer Amon Tobin, a sample's "point of origin" and the traceability of the original context are important factors when selecting material:

For me it's just important to use sounds that have been used in a different musical context previously. I've been really into that idea since my first record when I got so excited by being able to take a sliver of something and maintain the momentum of that snapshot. The idea of having a sound that doesn't have a 'point of origin' seems slightly flat (cited in Mackintosh 2003: 104).

When describing a sample's "point of origin", Tobin is referring to the mimetic qualities and cultural location of the sound rather than any gestural cause. Furthermore, his use of the term "snapshot" to describe the sampling process highlights one of the main reasons why people get so excited about sampling. Like photography, video and forms of digital art in general, sampling uses technologies that facilitate an ease of reproduction to a degree that can create a significant tension between what is technically achievable and what is ethically and legally permissible. ${ }^{29}$

\section{CAtegory (B): Loops AND Phrases}

\section{LOOPS AND PHRASES: SELF-REFERENTIAL MUSICAL ELEMENTS}

The first sub-category includes sampled musical phrases and loops that are self-referential. The presence of sampled material is unknown to the listener, which could be by designachieved through editing and processing, ${ }^{30}$ or through the obscurity of the source-or simply fortuitous, and consequently authorship of the material is attributed to the borrower. 
In this category, source recognition is irrelevant, with material selected purely for its musical content. For example, the use of a vocal phrase sampled from "You're No Good For Me" (1987) by Kelly Charles in the Prodigy's "No Good (Start The Dance)" (1994) does not require the listener to recognise the sample; ${ }^{31}$ it functions as a vocal element, irrespective of the source. Similarly, the vast range of borrowed melodic material, bass patterns, percussion loops and track segments used within EDM may also be included in this category. It is important to note, however, that the pervasiveness of certain samples (for example, the "funky drummer" loop ${ }^{32}$ ) has resulted in a situation in which previously unknown material has become referential through familiarity (Sanjek 1994). Such samples are located in the third sub-category.

\section{LOOPS AND PHRASES: REFERENTIAL NON-MUSICAL MATERIAL}

The second sub-category involves a referential or mimetic use of non-musical material. Here we can place cultural or environmental samples that do not have a conventional musical function, but are used for their associative, narrative or symbolic qualities. This includes real-world sounds like the ocean waves flowing through Goldie's "Sea of Tears" (1995) or the exotic animals of "It Began In Afrika" (2001) by the Chemical Brothers. Additional examples might include cultural signifiers, such as elements sourced from video games, radio, film and TV (dialogue, sound effects, etc.), the sound of church bells or a notable public event (for instance, the political speech delivered by John Major and sampled by Orbital on "Sad but New" (1995))..$^{33}$

Extensive material of this kind should be located within the corresponding sub-group of category C. However, it is possible to create the illusion of a more extended extract through the repetition of shorter overlapping samples or cross-fade looping. ${ }^{34}$ This is particularly effective when the loop points are masked by other textural elements (the Goldie example above appears to work in this way).

\section{LOOPS AND PHRASES: TRANSCONTEXTUAL MUSICAL ELEMENTS}

The third sub-category contains sampled material that continues to have a musical function, but in which a referential aspect is also present. This is a dual or "transcontextual" 35 use of sampled material, where it is intended for the listener to appreciate abstract musical properties, while simultaneously recognising the source of the sample and its extra-musical associations. Here we can locate cultural sounds (for example, samples of non-western music), or elements from popular music that are used either as prioritised quotations, or as musical components complete with extra mimetic associations. In the following example, the Chemical Brothers discuss the use of Moroccan music ${ }^{36}$ and accompanying cultural associations (a sample from "Just Tell Me The Truth" by Najat Aâtabou) in the context of their electronic track "Galvanize" (2005): 
That is a sample from a Moroccan record ... Everyone thinks it is Indian, but it is from Morocco. Someone gave me this record from the Ellipsis Arts label; it is amazing - so much energy and so raw. We brightened up the sound a bit and made it more aggressive, but it had so much power to begin with. The idea was to put it with this high-tech sound and get the worlds to collide. That is pretty much the sound that was on the record, backed up with a bit more oomph via effects (Rowlands cited in Micallef 2005).

Some sample-based tracks are saturated with borrowed elements that are easily recognisable. This includes the collage-based work of Bomb the Bass, Coldcut, M/A/R/R/S, S'Express and their contemporaries, as well as music that uses a limited number of samples combined with original components (for example, music by Leftfield or Underworld). In either case, the authorship of sampled material may not be acknowledged by the borrowing artist. In addition, there are samples that, although previously unknown, have become referential through frequent and widespread use (Sanjek 1994). For example, the "amen" ${ }^{37}$ drum loop has been used as a jungle signifier in experimental sub-genres of EDM, where it is subjected to complex and radical manipulations, often conducted with satirical intent. Such an example demonstrates how the selection and incorporation of borrowed elements within EDM may be driven by extra-musical factors. In this respect, there is a resonance with the goals of hip-hop musicians who seek "cultural literacy and intertextual reference" through the incorporation of recognisable material (Rose 1994: 89). According to Tricia Rose, hip-hop artists highlight sampled material and challenge the listener to locate the origin of the various musical and lyrical components (Rose 1994: 89). The educational value of incorporating recognisable material in this way has been noted by EDM musicians such as Tim Simenon (Bomb the Bass), who suggests that the music of James Brown would be unfamiliar to younger generations if it was not for the proliferation of his material within hip-hop (cited in Goodwin 1990). While sample-based EDM offers a similar challenge to listeners, there have been instances where the inclusion of well-known material in a new release has been revealed in advance of the music. In this way, the presence of familiar material is highlighted and used as a promotional tool to generate audience interest in the music prior to its availability. This was the case with the Prodigy's recontextualisation of "Thriller" (1982) by Michael Jackson, in "The Way It Is" (2004), ${ }^{38}$ which was highlighted during pre-release publicity ${ }^{39}$ of their fourth album, Always Outnumbered, Never Outgunned (2004). The inclusion of such well-known material gives rise to a situation in which the sample's source becomes cross-referential, with the original music evoking an image of the material in its recontextualised environment. This phenomenon can also be observed in popular music that incorporates samples as signifiers, seen for example in Robbie William's borrowing from "You Only Live Twice" (1967) by John Barry, in "Millennium" (1998), and Madonna's incorporation of sampled material from "Gimme, Gimme, Gimme (A Man After Midnight)" (1979) by Abba, in the track "Hung Up” (2005). 


\section{Category (C): Larger Elements}

\section{LARGER ELEMENTS: MULTI-TRACK PARTS AND A CAPPELLAS}

The first sub-category includes individual vocal or instrumental parts such as commercially released a cappellas and multi-track stems produced for DJ performance and remix purposes. ${ }^{40}$ Material may also be sourced from an existing track using specialised software tools and "unmixing" techniques (phase cancellation, spectral editing, equalisation, channel separation, etc.) to isolate and extract individual textural elements for subsequent sampling. This is standard practice within remix and mashup culture, ${ }^{41}$ where borrowed vocal and instrumental parts are used in a variety of ways, from detailed integration to superposition with minimal editing. Sampled elements may be extracted from a mono or stereo recording, or sourced from a multi-channel mix in which instrumental parts are located in specific audio channels and isolated more easily. By converting a multi-channel mix into separate mono files, it is possible to apply additional unmixing for thorough separation of the material. A related strategy involves the use of $\mathrm{MOGG}^{42}$ files sourced from music video games like Rock Band (2007) - these hold the multi-track parts of many established and well-known tracks that can be individually accessed through software such as Audacity ${ }^{43}$ (Mogg Files 2010).

It is possible to make a distinction between parts that are self-referential-as a result of editing or the obscurity of the sample-and those that are chosen for their referential or associative qualities (transcontextual). The recorded solo performance offers a potential source for both types of material. Here, we can cite the Fatboy Slim track "Sunset (Bird of Prey)" (2000) (with vocals sampled from the Jim Morrison poem "Bird of Prey" (1969)) and Moby's "Natural Blues" ${ }^{44}$ (1999) (based on a field recording of the African-American singer Vera Hall by the ethnomusicologist Alan Lomax). Furthermore, while both of these examples feature a substantial use of pre-recorded vocals, they demonstrate alternative approaches to the incorporation of such material, with the former based on the extraction and rearrangement of individual phrases, and the latter containing entire sections of unaltered material. The following quote from Future Loop Foundation describes an even more elaborate treatment:

There's a vocal part from a live session that I'd recorded and Michael had this idea for it which involved chopping the part up, de-breathing the parts, re-ordering them, taking lines out, adding syllables, putting a massive reverb on them, sampling them with the reverb, reversing them all so that there was a tail-out reverb at the start, adding another forward reverb, and finally re-reversing the whole thing so that there was a tail-in and tail out reverb on the final part (Barrott cited in O’Sullivan 1999: 64).

\section{LARGER ELEMENTS: EXTENDED EXCERPTS}

In the second sub-category we can locate extended excerpts of a complete track, including passages, sections and other significant material. A sample of this kind may be incorporated 
as an unaltered extract that is inserted or juxtaposed against other sections of the work, or combined with additional elements (especially drums and percussion) to create a new arrangement. In the second approach, the sample may form part of a sequence of extracts from the same source that are reordered to produce an alternative structural trajectory. This can be heard, for example, in "Wham! Bar" (2007) by Mint Royale, which uses substantial sections of "Wham Rap! (Enjoy What You Do)" (1982) by Wham.

An extended passage or section is likely to have a transcontextual function, due to the size of the material and the increased probability of recognition. It may be used as a cultural signifier, or contrasted stylistically with the original elements of a production, as in the classical interlude of Tiesto's "Athena" (2004) (an electronic "interpretation" ${ }^{45}$ of Albinoni's "Adagio in G minor"), and the James Bond inspired "On Her Majesty's Secret Service"46 (1997) by David Arnold and the Propellerheads.

\section{LARGER ELEMENTS: EXTENSIVE, REFERENTIAL NON-MUSICAL MATERIAL}

The third sub-category deals with extensive non-musical material that has a referential or mimetic function. It includes extended field recordings, foley sound, dialogue and other samples from category two that are significantly increased in length. Here we might locate a sampled answer phone message or recorded interview (like the extract of Rickie Lee Jones incorporated within the Orb's "Little Fluffy Clouds" ${ }^{47}$ (1990)), a documented public event (such as the religious sermon delivered by Reverend W. Leo Daniels and sampled by Fatboy Slim on "Drop The Hate" (2000)), or a recorded real-world environment (the city soundscape ${ }^{48}$ documented by Daft Punk in "Da Funk" (1997)). In the introduction to "High Rise" (1991) by Orbital, the presentation of a field recording with minimal editing highlights a connection to the "anecdotal" electroacoustic music of Luc Ferrari (and related practices) in which environmental recordings are sometimes used with little modification. At the same time, we can identify contrasting examples of recorded space, including a common form of "spatial simultaneity" (Smalley 1997) in which a remote space (such as an outdoor atmosphere) is layered behind the proximate components of a track (non-reverberant bass, drums and percussion): "sometimes it's like finding the perfect stereo atmosphere on a mini disc [sic] that will complement a mix, and that involves getting out of the house for once" (Clark cited in Barcode Magazine 2008).

\section{LARGER ELEMENTS: COMPLETE TRACKS}

The fourth sub-category consists of complete tracks or instrumental versions that are used as macro-level material-a practice that has become more widespread since the introduction of audio-based performance and production software such as Ableton Live. ${ }^{49} \mathrm{~A}$ common application is the "A vs. B" (or "A + B") mashup, in which a complete track or instrumental is combined with a different and often contrasting a cappella. The success of this approach is dependent, to a large extent, on the characteristics and compatibility of the chosen source materials, with integration of the components usually achieved through manipulation of 
large-scale musical parameters (such as tempo and key) and basic timbral modification (equalisation and filtering). An example is "Toca's Miracle" (2000), which brings together the a cappella of CoCo's "I Need A Miracle" (1996) and the synthetic-driven instrumental "Toca Me" (1999) by Fragma. The track was initially created by DJ Vimto as an unauthorised bootleg for DJ performance (Inglis 2000), but owing to its popularity an official version was later produced by Raymon Zenker (Fragma). In an interview with Sound On Sound magazine, Zenker revealed how the components were combined with little additional editing, consisting of minor sectional reorganisation and adjustment of the vocal tempo and pitch (Inglis 2000: 38):

The original vocals were at $126 \mathrm{bpm}$, so I had to timestretch them to 135 , I think, and I had to change the tuning a bit, it was a little bit out of tune, and then it fitted .... It was only about 50 or 30 cents out, just a bit (cited in Inglis 2000: 38).

Recognition of the source and context is not required in this case, ${ }^{50}$ but the origin of the material can play an important role: for artists like Mark Vidler (Go Home Productions), the mashup represents an opportunity to unite musicians from different times and places (Doyle 2004b), resulting in a "simulated" collaboration, "that did not, strictly speaking, ever take place" (Gunkel 2008: 498).

\section{CATEgory (D): Transformed MATERIAL}

The processing capabilities of the sampler have allowed EDM musicians to explore a more abstract soundworld ${ }^{51}$ (akin to that of electroacoustic composers) in which causal identity is removed, and the meaning of sounds is deduced by energy profile and morphology alone. ${ }^{52}$ At the same time, referential or causal information may provide a basis for a sound transformation in which meaning is drawn from the manipulation of a recognisable source. ${ }^{53}$ The following category is concerned with such samples that are transformed in terms of their spectral and morphological content (their "spectromorphology" ${ }^{4}$ (Smalley 1986 and 1997)), resulting in ambiguous or new material. Other types of transformation (for example, a reordering of the structural trajectory, or acts of "theft" which transform the meaning of sampled materials by surrounding them with "an aura of illegality" (Metzer 2003: 172)) $)^{55}$ are not considered, and should be included in the relevant sub-group of categories A, B, or C.

In describing the characteristics and processes of sample transformation, the EDM analyst may profit from Denis Smalley's article "Defining Transformations", which introduces terminology and a categorisation system based on "temporal relations" (Smalley 1993: 279). The following useful distinction is provided:

In attempting to define transformations we have to adopt two contrasted strategies which are directly related to the type of base identity. The first, linked to identifiable, transcontextual, source-causes, I shall call source-bonded transformation. The second, where extrinsic links and source-causes are imagined, imaginary, or seem so remote 
that they appear nonexistent, I shall call spectromorphological transformation. Sourcebonded transformation is source specific; spectromorphological transformation is source freed, more free-floating. In between the two are varying degrees of source inference and suggestion; we cannot say where one becomes the other (Smalley 1993: 282).

It is important to note that the two types (source-bonded and spectromorphological) "may collaborate", and that "in theory all transformation is created by spectromorphological means" (Smalley 1993: 285 and 282).

\section{TRANSFORMED MATERIAL: SHORT, ISOLATED FRAGMENTS}

The first sub-category includes short, isolated fragments that are transformed to create ornamental effects, or percussive or glitch-based sounds for use within a rhythmic pattern or stream. Here, the source bonding ${ }^{56}$ is removed, and the sound is used as something else. This approach has been encouraged by the increasingly sophisticated sound processing capabilities of sampling drum machines and sequencers, and the availability of software tools for conducting complex sound transformations. ${ }^{57}$ It is important to note, however, that such transformations are no longer confined to the studio environment and commonly appear during interactive electronic performances. Due to the increased power and stability of laptop technology, there is a growing practice of "live" sampling in which sounds are captured during performance and used as a basis for real-time composition and improvisation. For example, the experimental EDM producer Tim Exile has constructed a performance and composition system ${ }^{58}$-based around a custom-designed Reaktor ${ }^{59}$ environment and selection of hardware controllers - that facilitates live sampling and transformation. This system allows for material to be sampled (or resampled) from a microphone or other input source and integrated in real-time as part of a composition/performance. In this way, Tim Exile will often sample and transform his own voice to create new percussion sounds, building rhythmic material in layers as a performance unfolds.

\section{TRANSFORMED MATERIAL: LOOPS AND PHRASES}

The second sub-category deals with the transformation of larger elements. This might involve a covert transformation, in which concealed elements are unknown to the listener, or take the form of a perceivable transition, moving from clearly identifiable material through to the ambiguous and unrecognisable. Examples of the latter may be found in the work of Fatboy Slim, such as the dynamic time-stretch ${ }^{60} /$ pitch-shift degeneration of the track "Rockafeller Skank" (1998), or the ring-modulated vocal transition between the tracks "Retox" and "Weapon Of Choice" on his third album Halfway Between The Gutter And The Stars (2000).

As many EDM musicians sample directly from vinyl, ${ }^{61}$ turntable techniques, such as "scratching" 62 are often used to transform the identity of borrowed material. Realtime sample transformation has become much more prevalent since the introduction of 
performance samplers and DJ-oriented equipment. For example, Yamaha's SU700 and Roland's SP-808 samplers, and effects processors, such as Korg's Kaoss Pad, included a range of built-in controllers optimised for real-time manipulation and, in particular, the emulation of DJ-style effects. More recently, the innovation of virtual DJ technology has provided a more immediate way in which to apply turntable effects to sampled material.

\section{TRANSFORMED MATERIAL: LARGER ELEMENTS}

The final sub-category is concerned with the transformation of larger samples, including non-musical elements, multi-track parts, extended excerpts and complete tracks. These are frequently transformed in live performance to produce alternative or remixed versions of previously released music. Studio-based examples also exist, such as the processed dialogue from the science fiction film Beneath the Planet of the Apes (1970), which forms the introduction to "Desert Storm" (1991) by Orbital.

A transformation of this kind may be undertaken for practical reasons and, in particular, for the generation of variant or contrasting source material. In this way, the transformation is conceived as a form of "batch processing" that is applied globally to an extended sample, and allows for the timbre or spectromorphology of the source to be altered prior to dissection and resampling. The process can be repeated using different transformation settings and parameters to produce large quantities of source material, with suitable phrases and loops extracted from the resulting collection.

A distinction may be made at the procedural level between material that is directly captured from the source and subsequently modified, and that which is passed through additional equipment (such as a guitar pedal, synthesizer or chain of signal processing effects) for transformation en route to the recording device. A wide variety of results may be obtained, from subtle enhancement and controlled signal degradation (for integration with other low fidelity sound sources, such as vintage synthesizers and drum machines) to more significant source-bonded and spectromorphological transformation. The aim may be to disguise or to significantly alter the sample, or to maintain recognisability while further "personalising" the material, as David Morley describes: "always sample through something, be it an ARP 2600, a compressor, a preamp or a radio. This'll make your stuff sound like your stuff" (cited in Future Music 1998: 110).

\section{CONCLUSION}

The proposed typology provides a framework for the discussion of sampling practices within EDM. It is intended to contribute to the emerging descriptive and analytical vocabulary for the study of the genre, in order to facilitate a better understanding of the aims, motivations and work of EDM musicians and lead to a more informed consideration of sample-based musics in general. The typology may be applied to the study of a single track, or used as a system of cross-reference for the comparison of materials, attitudes and working methods between individual tracks, albums, sets, artists, genres or time periods. In this way, it allows 
for a broader consideration of sampling across the spectrum of EDM and, in particular, the identification of different origins and narratives, in terms of aesthetics and creative practice.

In addition, the typology demonstrates how the analytical and theoretical vocabulary of electroacoustic music can serve as a useful and effective resource for the EDM analyst. This is particularly important, as the lack of an established language for the study of EDM is problematic. Specific tools and methodologies have started to appear, but many important technical and theoretical aspects have yet to be explored or await further consideration, including, for example, the formalisation of methods for sound categorisation (the current article seeks to make a significant contribution in this area) and the development of appropriate forms of notation to cover the vast array of sonic and musical materials encountered within the genre. Similar issues have been tackled within the field of electroacoustic music, and the solutions and strategies developed there may help to answer some of the questions presently faced by the EDM analyst.

The electroacoustic language used within the typology highlights certain similarities between the sound materials, compositional techniques and production technologies of the two genres. It also suggests that the analytical and theoretical vocabulary of electroacoustic music may be widely applicable and relevant to various forms of EDM. A few potential applications might include: the use of Smalley's "spatiomorphology" to help describe contrasting "spatial settings" or the manipulation of "spatial perspective" in a production, mix or performance (1986 and 1997); Wishart's concept of the "sonic landscape" (1986), as an aid to the interpretation of different sonic environments within a track, set or series of related works (for instance, the surreal transition between "Skylined" and "Claustrophobic Sting" (1994) by the Prodigy (real and unreal objects/unreal space (Wishart 1986)); or Landy's "framework for the categorisation of sound transformation" (1991), used in relation to sound processing and ideas concerning "landscape" and "transcontextuality" (the transformation of a sample's meaning or context (Landy 1991)). Beyond these initial suggestions there are many other possible applications for the electroacoustic device, although the EDM analyst will need to be selective in choosing an appropriate tool or methodology for the work under consideration; for example, "spectromorphology" (Smalley 1986 and 1997) will be helpful when applied to the selected output of some EDM artists (those working with more abstract sound materials), but less effective when applied to others (those working with materials and structures that are essentially "instrumental" in nature or origin, or chosen for their cultural significance).

In conclusion, it is hoped that the examples offered by the typology will stimulate further interest in the analytical and theoretical vocabulary of electroacoustic music, as the descriptive and investigative possibilities afforded by this language ${ }^{63}$ could contribute to a better understanding of the sonic and musical aspects of EDM. For those wishing to pursue these ideas, the online repository for electroacoustic music analysis (oREMA) ${ }^{64}$ offers a useful starting point, including a range of example analyses and information about available tools and methodologies, while the electroacoustic resource site (EARS $)^{65}$ provides an online bibliography and a glossary of key terms. 


\section{ACKNOWLEDGEMENTS}

I am extremely grateful to both the AHRC for supporting the project, and to Mike Vaughan for his invaluable thoughts and advice. Sincerest thanks to Simon Emmerson, Sohrab Uduman and Rajmil Fischman for commenting on various versions of the typology. Thanks also to the Guest Editors Ed Montano and Simon Zagorski-Thomas, and the anonymous reviewers at Dancecult for their helpful suggestions.

\section{NOTES}

1 The typology was first conceived in 2008 as part of a larger research project (see Ratcliffe 2012). The classification system has since been significantly revised and expanded.

2 This is a process in which audio is taken from a pre-existing source and used as compositional material within a new work.

3 The different categories identified by Cutler (2000), Goodwin (1990), Porcello (1990) and Sanjek (1994) can be summarised as follows: 1 . Sampling embedded within devices such as sampling drum machines and synthesizers. 2. Creative practice that demonstrates an eclectic approach to musical sources and their juxtaposition. 3. Practices that "have made an aesthetic out of sampling ... and in some cases, a politics out of stealing" (Goodwin 1990: 271). 4. Sampling that explores the potential for sonic exploration, involving the appropriation, manipulation and transformation of materials as sound-sources (for example, during studiobased acousmatic composition or as raw input to a performance). 5. The use of sampling within remix practices.

4 Remix materials usually consist of samples extracted from the original track. These may be categorised according to the classification system proposed.

5 "In this article, the term EDM is used more broadly, encompassing both mainstream forms, such as drum 'n' bass and techno, and experimental sub-genres, such as breakcore and IDM" (Ratcliffe 2011:235).

6 This description is derived from Cox ("isolated sound fragments") (Cox 2007).

7 This could be melodic, harmonic, or rhythmic etc.

8 In the sense that something extra-musical is signified or recognition of the original context is intended.

9 For example, a Roland TR-808 bass drum sample is often used to provide extra sub-bass frequencies when layered with an existing kick drum.

10 Sampled atmosphere may include: ambience or reverberation sampled from an existing work, background noise (including environmental recordings), and sonic artifacts introduced via the recording and production process (for example, analogue tape "hiss" or vinyl noise (the "phonograph effect" (Katz 2010: 155))). In the example above, DJ Shadow documents an approach whereby sampled atmosphere is used to add authenticity in the simulation of a recorded live performance. EDM musicians frequently adopt this strategy when creating new material from individual fragments; for example, Fatboy Slim described the use of sampled "air" to conceal the edits in a reconfigured drum loop (cited in Scott 2001: 100). 
11 This is where we can detect a physical human gesture as the cause or trigger for the sound (such as playing a drum pad or keyboard).

12 For example, the Future Sound of London would record themselves "hitting railings for percussive sounds" (Cobain cited in Buskin 2006: 58).

13 For more information, see: <http://www.propellerheads.se/products/recycle/> (accessed 28 November 2011).

14 Breakcore is "an extreme, hyper-accelerated form of EDM, often involving the use of complex, edited breakbeat patterns. The most notable exponent of the genre is Venetian Snares" (Ratcliffe 2011). According to Matt Earp of XLR8R, "Scud and Nomex tracks like "Total Destruction" helped create the blueprint for much of breakcore's sound, a high-bpm mash-up of hyperkinetic, post-jungle breaks, feedback, noise, and Jamaican elements paired with a devil-may-care attitude towards sampling that pulls from the broadest spectrum of styles (hip-hop, rock, industrial, pop, and beyond)" (Earp 2006). See < http://www.xlr8r.com/ features/2006/05/breakcore-live-fast $>$ (accessed 28 November 2011).

15 Sound Forge is a software application for digital audio recording, editing, and mixing. See: $<$ http://www.sonycreativesoftware.com/soundforgesoftware $>$ (accessed 28 November 2011).

16 According to the sleeve notes, the source material consisted of video footage provided by Greenpeace, while the track itself was inspired by the "video sampling" of artists such as Steinski: "all sound sources are linked to their video sources. Whole rhythms have been painstakingly edited out of individual beats and frames" ("Timber" 1998 single release).

17 Emmerson defines mimesis as "the imitation not only of nature but also of aspects of human culture not usually associated directly with musical material” (1986:17).

18 Emmerson describes two types of mimesis: “timbral' mimesis is a direct imitation of the timbre ('colour') of the natural sound, while 'syntactic' mimesis may imitate the relationships between natural events; for example, the rhythms of speech may be 'orchestrated' in a variety of ways" (1986: 17-18).

19 The success of this approach depends to a certain degree on the timbral distinctiveness of the sample: “. . a a fan can recognise a hit from a ten-millisecond burst” (Oswald 2004: 133).

20 DJ Shadow's sample-constructed debut album Endtroducing (1996) was created using limited technical resources, consisting of an Akai MPC 60 MK II drum machine, a turntable and an Alesis ADAT digital recorder (DJ Shadow cited in Keyboard 1997). Endtroducing is recognised by Guiness World Records as the first, entirely sample-based album (see < http:// www.guinnessworldrecords.com/records-1000/first-album-made-completely-from-samples/> (accessed 28 November 2011)), although this decision has been questioned due to the apparent inclusion of live scratching and recorded vocals (Doran 2010).

$21 \mathrm{MPC}$ is the model designation for a range of a sampling drum machine/sequencers, originally designed by Roger Linn and released by Akai from 1988 onwards (for instance, the MPC 60, MPC 2000, and MPC 3000). These instruments are favoured for sample-based hip-hop and EDM due to both the design of the user interface (featuring drum pads for real-time programming) and idiomatic performance characteristics (such as the swing quantisation algorithm).

22 This is a synthesis system in which an oscillator-generated waveform (the sound source) is supplemented or replaced with a stored audio waveform. 
23 It has been increasingly common for the "reese" bass sound to be imitated (and adapted) using synthesis rather than direct sampling. Tutorial patches have been demonstrated, for example in Computer Music magazine (see Sub Focus 2007).

24 As described in the sleeve notes ("Timber" 1998 single release).

25 In addition to the shorter sound fragments, there are various phrase-level samples (for instance, during the breakdown). These should be located in category B.

26 For example, the Future Sound of London "had big bags of samples and dubs and noises" and would "sprinkle them over things" (Dougans cited in Buskin 2006: 58).

27 To borrow from the electroacoustic resource site: "mimetic discourse refers to the signifying potential of referential or extrinsic attributes of sound" (Ears 2013).

28 A more recent example was the use of rave signifiers on the Prodigy's fifth studio album, Invaders Must Die (2009).

29 A consideration of related ethical and legal issues is beyond the scope of this article. For further discussion see, for instance, Porcello (1991) and Schumacher (1995).

30 It is important to note that musical and spectral information from the original sample will be retained, as opposed to radical spectromorphological change, resulting in new material. Such transformations are included in category $\mathrm{D}$.

31 A useful online resource is available at <http://www.whosampled.com/ > (accessed 28 November 2011). This is a comprehensive comparative database for the identification of borrowed material in sample-driven music (including remixes and cover versions). Through the use of embedded audio files (invariably linked via YouTube), users may directly compare a sample-based track with original source material. It is an ongoing community-driven project, with users actively involved in the identification process.

32 This is a funk breakbeat performed by Clyde Stubblefield on the James Brown track, "Funky Drummer" (1970). It is one of the most commonly used samples in popular music, and regularly found in EDM genres, such as jungle, drum 'n' bass and big beat. For more information on sampled drum loops, see for example, the online database available at <http://www.junglebreaks.co.uk/> (accessed 28 November 2011). This site reveals the most frequently sampled breakbeats within jungle and drum ' $n$ ' bass, providing detailed discographic information for each sample (original source and subsequent use). A WAV file of each sample is also available for online listening or download.

33 I am referring specifically to the sampled voice and applause heard at the start of the track. Additional (and substantial) extracts of the speech were used on the live version broadcast on BBC Radio 1 in 1995 (see Loopz n.d.).

34 This is a facility offered by some hardware and software samplers. It allows the user to crossfade the start/end points of a looped sample, resulting in a smoother and more continuous sound.

35 The concept of transcontextuality is taken from Smalley (1992), who originally borrowed from the literary theorist Linda Hutcheon (1985). According to Smalley, a "transcontext" occurs when "the composer intends that the listener should be aware of the dual meanings of a source. The first meaning derives from the original, natural or cultural context of the event; the second meaning derives from the new, musical context created by the composer" (Smalley 1992: 542). In this article, the term is reserved for the description of recognisable musical 
elements (loops, phrases and larger elements), while "recontextualisation" is preferred in relation to referential non-musical samples.

36 The sampling and appropriation of non-Western music by Western musicians has been discussed by Hesmondhalgh (2000).

37 The "amen" breakbeat is sourced from the track "Amen, Brother" (1969) by The Winstonsan instrumental cover version of a standard gospel song (Shapiro 2000: 153). The "amen" loop has been sampled in various genres (including hip-hop), but is most commonly known as a convention of jungle and drum ' $n$ ' bass. Here it is utilised at a significantly faster tempo, which was initially achieved through the technique of pitch-shifting, producing a distinctive sound that has become part of the sample's identity. The "amen" is still used in contemporary drum 'n' bass and as a signifier in experimental forms of EDM, such as breakcore and IDM. A notable example is the music of Amen Andrews (Luke Vibert) who has produced a body of work based on the manipulation of the "amen" sample (for example, the album Amen Andrews Vs. Spac Hand Luke (2006)).

38 The material from “Thriller” was completely recreated, as Rod Temperton ("Thriller's" composer) would not allow direct use of the original recording (see DJ Ron Slomowicz n.d.). For more on "sample recreation" - a process in which an unauthorised sample is replicated both sonically and musically in order to function as a replacement (free of the mechanical copyright) — see Inglis (2003).

39 For example, the feature article by Martin James in Future Music magazine (James 2004).

40 In the form of sample CDs and DJ tools (such as the Defected "Accapella" series), or sample packs made available through online music stores or remix competitions (some of the most popular online sources include Beatport (<www.beatport.com/ $>$ ), Indaba Music (<www. indabamusic.com/ $>$ ), and the now defunct Back 2 You).

41 For more on the audio mashup, see McGranahan (2010).

42 This is a container file holding multiple OGG files (Mogg Files 2010).

43 Audacity is an open-source application for audio recording and editing. It is freely available from: <www.audacity.sourceforge.net/> (accessed 9 June 2013).

44 This track is taken from the album Play (1999), which is notable for the sampling of historical field recordings, containing music by African-American vocalists. For further discussion and analysis in relation to "cultural borrowing", see Hesmondhalgh (2006).

45 As described in the album sleeve notes (Parade of the Athletes (2004)).

46 It should be noted that the orchestral samples were performed and recorded especially for the project, as opposed to sampling directly from the original soundtrack. A related practice involves the use of session musicians to generate unique source material through directed performance or improvisation. Such methods were employed by Daft Punk during the construction of their most recent album Random Access Memories (2013), with a number of legendary session musicians contributing to the record. For more information, see Tingen (2013).

47 This is a seminal sample-based production. A partial analysis and graphical transcription is provided by Holm-Hudson (1997). For information concerning the production and context of the track, see Buskin (2011). 
48 The sample continues for the first twenty-five seconds of the track, moving from foreground to background.

49 For more information see: < https://www.ableton.com/en/> (accessed 1 June 2013).

50 The vocal functions as a self-referential melodic and textural component-something that Zenker felt was lacking from the original version (Zenker cited in Inglis 2000).

51 The reasons for carrying out an act of transformation may not only be artistic, but may also arise from a necessity to conceal the source of an "unauthorised" sample. This is especially true if the material has been taken from a widely recognised source and the probability of identification is increased.

52 For an extensive discussion see Smalley (1986 and 1997).

53 Including, but not limited to: forms of non-musical material (the filtered rainfall in Orbital's "I wish I had Duckfeet" (1994)); individual parts and phrases (the "revelatory transformation" (Smalley 1993) of Daft Punk's "Daftendirekt" (1997) or the obliterated drum loop of the Chemical Brother's "Block Rockin' Beats (the Micronauts Remix)" (1997)); or an extract of a complete track (the descending, multi-stage, pitch-shift of "Axis" (1993) by DJ Solo (featuring DJ Devine) in the Daedelus remix of "Down" by Sepalcure (2011)).

54 This term is sourced from electroacoustic theory. Smalley "developed the concepts and terminology of spectromorphology as tools for describing and analysing listening experience. The two parts of the term refer to the interaction between sound (spectro-) and the ways they change and are shaped through time (-morphology). The spectro- cannot exist without the -morphology and vice versa: something has to be shaped, and a shape must have sonic content" (Smalley 1997: 107).

55 While some musicians negotiate the issue of copyright through sample clearance, others wish to be provocative by deliberately highlighting the use of unauthorised material. This may be a political gesture or the "illegal" may have a particular appeal to their fanbase.

56 This term was introduced by Smalley to describe "the natural tendency to relate sounds to supposed sources and causes, and to relate sounds to each other because they appear to have shared or associated origins" (Smalley 1997: 110).

57 Examples include hardware devices (such as the Elektron Machinedrum SPS-1UW and Octatrack), software instruments and plug-in effects (such as Native Instruments Battery), and also a range of hybrid instruments that combine software editing with a hardware interface (for example, Native Instruments Maschine and Artruria's Spark).

58 For further information, see Tim Exile's video demonstration: $<$ http://www.youtube.com/wat $\underline{\mathrm{ch}} \mathrm{v}=9 \mathrm{r} 38 \mathrm{r} 3 \mathrm{BIgew} \&$ feature $=$ relmfu $>($ accessed 28 November 2011).

59 Reaktor is a virtual studio and graphical programming environment developed by Native Instruments. It allows users to design and construct their own software instruments, ranging from synthesizers, samplers and sequencers to effects processors and more esoteric devices. There is an extensive online library in which users may share their creations and download additional instruments. For further information see $<$ http://www.native-instruments.com/\#/ en/products/producer/reaktor-5/> (accessed 28 November 2011).

60 "Time-stretching" is a digital processing technique in which the length of a sample may be compressed or expanded without affecting the pitch or timbre. Time-stretching was also used as a colouristic device by jungle and drum 'n' bass musicians, especially when working with 
percussion samples (for instance, on the track "Sound Control" (1994) by Randall and Andy C) and vocal material (for example, on "BOOYAAA! (Open Your Mind)" (1994) by Amazon II and "Screwface 2" (1994) by Dubtronic), with the effect becoming common practice through inclusion on a countless number of tracks. According to Jon Bickle (inMusic Brands), the $S 950$ was the first Akai sampler to implement time-stretching. 15,000 units were produced from 1988-93 (Jon Bickle email to author, 2 August 2013).

$61 \mathrm{EDM}$ musicians have been known to press samples onto vinyl for manipulation using scratching and other turntable techniques (for example, Coldcut (see Robinson 1997)). It should be noted, however, that this practice has become somewhat obsolete since the advent of virtual DJ technology and emulative turntable effects.

62 This is a technique in which a vinyl record is pushed back and forth against the stylus to create new rhythmic and sonic material. For an in-depth discussion and analysis of scratching techniques, see Hansen (2002).

63 Ideally, used as part of an expanded set of resources. As I have suggested elsewhere, the diverse nature of the EDM genre requires "an adaptable and multifaceted approach to analysis", which might also include, for example, elements drawn from popular music (Ratcliffe 2013).

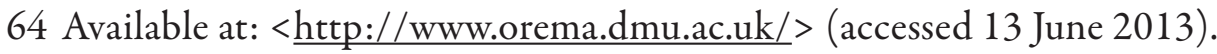

65 Available at: $<$ http://www.ears.dmu.ac.uk/ $>$ (accessed 13 June 2013).

\section{REFERENCES}

Barcode Magazine. 2008. "Chris Clark Interview."

$<$ http://www.barcodezine.com/Chris\%20Clark\%20Interview.htm> (accessed 12 June 2013).

Buskin, Richard. 2006. “The Future Sound of London: 'Papua New Guinea." Sound On Sound. November: 54-59.

- - 2011. "The Orb: Little Fluffy Clouds." Sound On Sound. August: $<$ http://www. soundonsound.com/sos/aug11/articles/classic-tracks-0811.htm > (accessed 13 June 2013).

Butler, Mark J. 2006. Unlocking the Groove: Rhythm, Meter, and Musical Design in Electronic Dance Music. Bloomington and Indianapolis: Indiana University Press.

Cox, Geoffrey M. 2007. "Largely a Work of Reference: A Creative Investigation into the Use of Borrowed Musical Materials in Contemporary Compositional Practice". Ph.D. Thesis (Composition), Huddersfield University.

Cutler, Chris. 2000. "Plunderphonics." In Music, Electronic Media and Culture, ed. Simon Emmerson, 87-114. Aldershot: Ashgate.

DJ Ron Slomowicz. n.d.. "Prodigy Interview." < http://dancemusic.about.com/od/ artistshomepages/a/ProdigyInt.htm $>$ (accessed 4 February 2012).

Doran, John. 2010. "Megadef: DJ Shadow Interviewed." The Quietus. 18 October: $<$ http://thequietus.com/articles/05112-dj-shadow-interview $>$ (accessed 4 February 2012).

Doyle, Tom. 2004a. "Matmos." Sound On Sound. May: 78-83.

- - . 2004b. "Bootleg Mixing: Richard X \& Mark Vidler." Sound On Sound. December. 
Earp, Matt. 2006. “Breakcore: Live Fast.” XLR8R. 20 June: <http://www.xlr8r.com/ features/2006/05/breakcore-live-fast $>$ (accessed 4 February 2012).

EARS: Electroacoustic Resource Site. n.d. "Aural Discourse and Mimetic Discourse." <http://www.ears.dmu.ac.uk/spip.php?rubrique181 > (accessed 13 June 2013).

Emmerson, Simon. 1986. "The Relation of Language to Materials”, in The Language of Electroacoustic Music, ed. Simon Emmerson, 17-39. Basingstoke: Macmillan.

Future Music. 1998. “Unique Sounds: Sonic Elements from the Stars.” July: 96-118.

Goodwin, Andrew. 1990. "Sample and Hold: Pop Music in the Digital Age of Reproduction." In On Record: Rock, Pop, and the Written Word, ed. Simon Frith and Andrew Goodwin, 258-73. New York: Pantheon Books.

Gunkel, David. J. 2008. "Rethinking the Digital Remix: Mash-ups and the Metaphysics of Sound Recording." Popular Music and Society 31(4): 489-510.

Hansen, Kjetil Falkenberg. 2002. "The Basics of Scratching.” Journal of New Music Research 31(4): 357-65.

Hesmondhalgh, David. 2000. "International Times: Fusions, Exoticism, and Antiracism in Electronic Dance Music." In Western Music and its Others: Difference, Representation, and Appropriation in Music, ed. Georgina Born and David Hesmondhalgh, 280-304. Berkeley: University of California Press.

_- - 2006. "Digital Sampling and Cultural Inequality." Social \& Legal Studies 15(1): 5375. Holm-Hudson, Kevin. 1997. "Quotation and Context: Sampling and John Oswald's Plunderphonics.” Leonardo Music Journal 7: 17-25.

Hutcheon, Linda. 1985. A Theory of Parody: The Teachings of Twentieth-Century Art Forms. London: Methuen.

Inglis, Sam. 2000. “Tracks: Recording Fragma's 'Toca’s Miracle.” Sound On Sound. September: 36-38.

- _ 2003 "Rinse: Steve Gibson \& Dave Walters: Recreating Samples”. Sound On Sound. September: 194-98.

James, Martin. 2004. “The Prodigy.” Future Music. September: 84-90.

Katz, Mark. 2010. Capturing Sound: How Technology Has Changed Music. Rev. Ed. London: University of California Press.

Keyboard. 1997. October: <http://www.solesides.com/winblad/shadowkeyboard1097.html> (accessed 4 February 2012).

Landy, Leigh. 1991. "Sound Transformation in Electroacoustic Music." Composers Desktop Project Quarterly. <http://www.composersdesktop.com/landyeam.html> (accessed 23 June 2013).

Loopz. n.d. "Online Orbital Discography." < http://www.loopz.co.uk/discog/ep/timesfly.html> (accessed 14 June 2013).

Mackintosh, Hamish. 2003. “Amon Tobin.” Future Music. September: 100-5.

McGranahan, Liam. 2010. "Bastards and Booties: Production, Copyright, and the Mashup Community." TRANS-Transcultural Music Review 14: < $\underline{\text { http://www.sibetrans.com/trans/a13/ }}$ bastards-and-booties-production-copyright-and-the-mashup-community> (accessed 8 June 2013).

Metzer, David. 2003. Quotation and Cultural Meaning in Twentieth-Century Music. Cambridge: Cambridge University Press. 
Micallef, Ken. 2002. "Chemical Brothers Interview." Remix. January: <http://planet-dust. laserjay.net/remix.htm> (accessed 4 February 2012).

- - - 2005. "Innovate or Evaporate." Remix. 1 February: <http://emusician.com/remixmag/ artists interviews/musicians/remix innovate evaporate/> (accessed 4 February 2012).

MOGG Files - Multitrack Digital Audio Format. 2010. "Is this the Future of Listening to Music? Think of it as a Multitrack MP3.” 13 December: < http://moggfiles.wordpress.com/> (accessed 9 June 2013).

O’Sullivan, Derek. 1999. "Killer Loop." Future Music. January: 60-64.

Oswald, John. 2004. "Bettered by the Borrower: The Ethics of Musical Debt." In Audio Culture: Readings in Modern Music, ed. Christoph Cox and Daniel Warner, 131-7. New York and London: Continuum.

Porcello, Thomas. 1991. “The Ethics of Digital Audio-Sampling: Engineers' Discourse”. Popular Music 10(1): 69-84.

Pytlik, Mark. 2002. “The Avalanches: Darren Seltmann and Robbie Chater.” Sound On Sound. November: 74-80.

Ratcliffe, Robert. 2011. "New Forms of Hybrid Musical Discourse: An Exploration of Stylistic and Procedural Cross-fertilisation between Contemporary Art Music and Electronic Dance Music." In Proceedings of the International Computer Music Conference 2011, 235-42. Huddersfield/San Francisco: ICMA.

- - - 2012. "New Forms of Hybrid Musical Discourse: An Exploration of Stylistic and Procedural Cross-fertilisation between Contemporary Art Music and Electronic Dance Music.” Ph.D. Thesis (Composition and Musicology), Keele University.

_- - 2013. "Analytical Précis of Chime by Orbital: Towards an Analysis of Electronic Dance Music." eOREMA 1. < http://dx.doi.org/10.3943/001.2013.04.0104 >.

Reynolds, Simon. 2010. Generation Ecstasy: Into the World of Techno and Rave Culture. New York: Routledge.

Robinson, Dave. 1997. “The First Cut is the Coldest.” Future Music. Special: 64-8.

Rodgers, Tara. 2003. "On the Process and Aesthetics of Sampling in Electronic Music Production." Organised Sound 8(3): 313-20.

Rose, Tricia. 1994. Black Noise: Rap Music and Black Culture in Contemporary America. Hanover and London: University Press of New England.

Sanjek, David. 1994. “"Don't Have to DJ No More”: Sampling and the "Autonomous" Creator." In The Construction of Authorship: Textual Appropriation in Law and Literature, ed. Martha Woodmansee and Peter Jaszi, 343-60. Durham and London: Duke University Press.

Schumacher, Thomas, G. 1995. “"This is a Sampling Sport”: Digital Sampling, Rap Music and the Law in Cultural Production." Media, Culture and Society 17(2): 253-73.

Scott, Danny. 1998. “The Men from U.N.K.L.E.” Future Music. October: 96-100.

_-_. 2001. “Stormin' Norman.” Future Music. March: 94-101.

Sellars, Paul. 2002. "Designer Label: Mike Paradinas and Planet Mu." Sound On Sound. May: 176-80.

Shapiro, Peter, ed. 2000. Modulations-A History of Electronic Music: Throbbing Words on Sound. New York: Caipirinha Productions. 
Smalley, Denis. 1986. "Spectro-morphology and Structuring Processes." In The Language of Electroacoustic Music, ed. Simon Emmerson, 61-93. Basingstoke: Macmillan.

_- _. 1992. "The Listening Imagination: Listening in the Electroacoustic Era." In Companion To Contemporary Musical Thought, ed. John Paynter; Tim Howell; Richard Orton; Peter Seymour, 514-54. London: Routledge.

- - . 1993. "Defining Transformations." Journal of New Music Research 22(4): 279-300.

-_- 1997. "Spectromorphology: Explaining Sound-Shapes." Organised Sound 2(2): 107-26.

Sub Focus. 2007. “The Ultimate Guide to Drum 'n' Bass.” Computer Music. August: 30-1.

Tingen, Paul. 2013. "Daft Punk: Peter Franco \& Mick Guzauski: Recording Random Access Memories." Sound On Sound. July: <http://www.soundonsound.com/sos/jul13/articles/daftpunk.htm > (accessed 1 August 2013).

Wishart, Trevor. 1986. "Sound Symbols and Landscapes." In The Language of Electroacoustic Music, ed. Simon Emmerson, 41-60. Basingstoke: Macmillan.

\section{DISCOGRAPHY}

Abba. 1979. Gimme, Gimme, Gimme (A Man After Midnight). Epic (7-inch): S EPC 7914. <http://www.discogs.com/ABBA-Gimme-Gimme-Gimme-A-Man-After-Midnight/ release $/ 429128>$.

Amazon II. 1994. BOOYAAA! (Open Your Mind). Aphrodite Recordings (12-inch): APH-11aa. $<$ http://www.discogs.com/Amazon-II-Booyaaa-Open-Your-Mind-Control-Yourself/ release $/ 55031>$.

Amen Andrews Vs. Spac Hand Luke. 2006. Amen Andrews Vs. Spac Hand Luke. Rephlex (CD):CAT 178 CD. $<$ http://www.discogs.com/Amen-Andrews-Vs-Spac-Hand-Luke-Amen-Andrews-Vs-SpacHand-Luke/release/716101>.

Barry, John. 1997. You Only Live Twice, on The Best OfJohn Barry: Themeology. Columbia (CD): 488582 2. <http://www.discogs.com/John-Barry-Themeology/release/465335>.

Brown, James. 1970. Funky Drummer. King Records (7-inch): 45-6290. $<$ http://www.discogs.com/James-Brown-Funky-Drummer/release/560161 >.

Charles, Kelly. 1987. You're No Good For Me. London Recordings (12-inch): LONX 153. $<$ http://www.discogs.com/Kelly-Charles-Youre-No-Good-For-Me/release/69452>.

Chemical Brothers, The. 1997. Block Rockin'Beats. Virgin Records (CD): CHEMSDX5. < http://www.discogs.com/Chemical-Brothers-Block-Rockin-Beats/release/58992>.

- - . 2001. It Began in Afrika. Virgin Records (CD): CHEMSD12. <http://www.discogs.com/Chemical-Brothers-It-Began-In-Afrika/release/75738>.

—- - 2005. Galvanize. EMI (CD): CHEMSDX21. <http://www.discogs.com/Chemical-Brothers-Galvanize/release/379091>.

Coco. 1996. I Need a Miracle. Greenlight Recordings (12-inch): GL004. < http://www.discogs.com/Coco-I-Need-A-Miracle/release/57473 >.

Coldcut and Hexstatic. 1998. Timber. Ninja Tune (CD): ZEN CDS65A. <http://www.discogs.com/Coldcut-Hexstatic-Timber/release/31015 $>$. 
Daft Punk. 1997. Homework. Virgin Records (CD): CDV 2821. < http://www.discogs.com/Daft-Punk-Homework/release/3235>.

-_- 2013. Random Access Memories. Columbia Records (CD): 88883716862.

DJ Shadow. 1996. Endtroducing. Mo' Wax Recordings (CD): MW059CD. <http://www.discogs.com/DJ-Shadow-Endtroducing/release/2876147>.

DJ Solo. 1993. Axis / Darkage. Production House (12-inch): PNT O50. <http://www.discogs.com/DJ-Solo-Axis-Darkage/release/80891>.

Dubtronic. 1994. Screwface 2, on The Ultimate Drum \& Bass Collection. Quality Price Music (CD COMP): QPMCD2/A. <http://www.discogs.com/Various-The-Ultimate-Drum-Bass-Collection/release/646962>.

Fatboy Slim. 1998. You've Come Along Way, Baby. Skint Records (CD): BRASSIC 11CD. <http://www.discogs.com/Fatboy-Slim-Youve-Come-A-Long-Way-Baby/release/2704>.

- - 2000. Halfway Between the Gutter and the Stars. Skint Records (CD): BRASSIC20CD. <http://www.discogs.com/Fatboy-Slim-Halfway-Between-The-Gutter-And-The-Stars/release/8045>.

Fragma. 1999. Toca Me. Positiva (12-inch): 12TIV-120. <http://www.discogs.com/Fragma-Toca-Me/release/27655>.

-_- 2000. Toca's Miracle. Positiva (12-inch): 12TIV-128. <http://www.discogs.com/Fragma-Tocas-Miracle/release/22695>.

Goldie. 1995. Timeless. FFRR/Metalheadz (CD): 828 646-2. < http://www.discogs.com/Goldie-Timeless/release/11681 >.

Jackson, Michael. 1982. Thriller. Epic (LP): EPC 85930. <http://www.discogs.com/Michael-Jackson-Thriller/release/1762968>.

Madonna. 2005. Confessions on a Dance Floor. Warner Bros (CD): 9362-49460-2. $<$ http://www.discogs.com/Madonna-Confessions-On-A-Dance-Floor/release/555652>.

Mint Royale. 2007. Pop is... Faith \& Hope Records Limited (CD/DVD): FH066CD. <http://www.discogs.com/Mint-Royale-Pop-Is/release/932683 >.

Mistabishi. 2009. Drop. Hospital Records (CD): NHS148CD. <http://www.discogs.com/Mistabishi-Drop/release/1661999) .

Moby. 1999. Play. Mute (CD): CDStumm172. <http://www.discogs.com/Moby-Play/release/2285>.

Morrison, Jim (with music by the Doors). 1995. An American Prayer. Elektra (CD): 7559-61812-2. $<$ http://www.discogs.com/Jim-Morrison-Music-By-Doors-An-American-Prayer/ release $/ 379362>$.

Orb, The. 1991. The Orb's Adventures Beyond the Ultraworld. Wau! Mr. Modo Records (CD): BLRDCD 5. <http://www.discogs.com/Orb-The-Orbs-Adventures-Beyond-The-Ultraworld/release/13265>.

Orbital. 1991. [Green Album]. FFRR (CD): 8282482. <http://www.discogs.com/Orbital-Orbital-Green-Album/release/143056>.

- —. 1994. Snivilisation. Internal (CD): 828 536.2. <http://www.discogs.com/Orbital-Snivilisation/release/4472771>.

- - . 1995. Times Fly. Internal (CD EP): LIECD23. <http://www.discogs.com/Orbital-Times-Fly/release/24599>. 
Prodigy, The. 1994. Music for the Jilted Generation. XL Recordings (CD): XLCD 114. < http://www.discogs.com/Prodigy-Music-For-The-Jilted-Generation/release/2719>.

- - 2004. Always Outnumbered, Never Outgunned. XL Recordings (CD): XLCD 183. $<$ http://www.discogs.com/Prodigy-Always-Outnumbered-Never-Outgunned/ release/313346>.

- - 2009. Invaders Must Die. Take Me to the Hospital (CD): HOSPCD001X. <http://www.discogs.com/Prodigy-Invaders-Must-Die/release/1660773 >.

Propellerheads, The. 1998. Decksandrumsandrockandroll. Wall of Sound (CD): WALL CD015. < http://www.discogs.com/Propellerheads-Decksandrumsandrockandroll/release/12037>.

Randall and Andy C. 1994. Sound Control. RAM Records (12-inch): RAMM 11. $<$ http://www.discogs.com/Randall-Andy-C-Sound-Control-Feel-It/release/1479>.

Reese. 1988. Just Want Another Chance. Incognito Records (12-inch): IR 111787. <http://www.discogs.com/Reese-Just-Want-Another-Chance/release/18335 >.

Sepalcure. 2011. Love Pressure Remixed. Hotflush Recordings (12-inch): HFRMX008. <http://www.discogs.com/Sepalcure-Love-Pressure-Remixed/release/3067409>.

Tiesto. 2004. Parade of the Athletes. Nebula (CD): nebcd9011. <http://www.discogs.com/Ti\%C3\%ABsto-Parade-Of-The-Athletes/release/330040>.

T. Power. 1995. The Mutant Remix - Rollers Instinct [remix by DJ Trace]. SOUR (12-inch): SOUR026. <http://www.discogs.com/TPower-Turquoise-The-Mutant-Remix/release/20722>.

Wham. 1982. Wham Rap! (Enjoy What You Do) - Special U.S. Remix. Inner Vision Records (7-inch): IVL A2442. <http://www.discogs.com/Wham-Wham-Rap-Enjoy-What-You-Do-Special-US-Re-Mix/release/1691509>.

Williams, Robbie. 1998. Millennium. Chrysalis (CD): CDCHS 5099. <http://www.discogs.com/Robbie-Williams-Millennium/release/37856>.

Winstons, The. 1969. Color Him Father / Amen, Brother. Metromedia Records (7-inch): MMS-117. < http://www.discogs.com/Winstons-Color-Him-Father-Amen-Brother/release/423956>.

FILMOGRAPHY

Post, Ted. Beneath the Planet of the Apes. 1970. USA: Twentieth Century Fox. $<$ http://www.imdb.com/title/tt0065462/>. (accessed 1 August 2013).

“Tim Exile Shows his Reaktor-Based Live Performance Setup”. 2009. Posted by NativeInstruments on YouTube. $<$ http://www.youtube.com/watch?v=9r38r3BIgew\&feature=relmfu $>$. (accessed 28 November 2011). 